\title{
Vortex-antivortex pair generation by an in-plane magnetic dipole on a superconducting film
}

\author{
V. N. Gladilin, ${ }^{1,2}$ J. Tempere, ${ }^{1}$ J. T. Devreese, ${ }^{1}$ W. Gillijns, ${ }^{2}$ and V. V. Moshchalkov ${ }^{2}$ \\ ${ }^{1}$ TFVS, Universiteit Antwerpen, Groenenborgerlaan 171, 2020 Antwerpen, Belgium \\ ${ }^{2}$ INPAC, K.U.Leuven, Celestijnenlaan 200 D, B-3001 Leuven, Belgium
}

(Received 30 June 2009; published 4 August 2009)

\begin{abstract}
We apply the time-dependent Ginzburg-Landau approach to describe the nucleation of vortex-antivortex pairs in a hybrid system, formed by a thin superconducting film with a single in-plane magnetic dipole on top of it, and to simulate the vortex dynamics in the presence of a direct transport current. At current densities, sufficient to depin vortices and antivortices from a magnetic dipole, it periodically generates vortex-antivortex pairs, producing vortex and antivortex streams which eventually coalesce into phase-slip lines with further increasing current. We demonstrate the possibility to efficiently control the generation rate and trajectories of emitted vortices by the applied current density and an additional weak homogeneous magnetic field.
\end{abstract}

DOI: 10.1103/PhysRevB.80.054503

PACS number(s): 74.78.-w, 74.20.De, 74.25.Qt

\section{INTRODUCTION}

Over the last decades, phenomena originating from the interplay of two mutually exclusive states of matter, superconductivity and ferromagnetism, combined in superconductor-ferromagnet hybrids, have attracted much attention in both experimental and theoretical studies (see reviews Refs. 1-3). Considerable efforts, fueled to a great extent by perspectives to improve the superconducting critical parameters, have been focused on vortex pinning in heterostructures, composed by planar superconductors and (sub) micron ferromagnetic dots (see Ref. 3 for a recent review). Due to the magnetic nature of the pinning centers, pinning effects can be significantly enhanced in those structures, ${ }^{4-7}$ which, in addition, provide a unique opportunity to tune field-induced superconductivity by changing the magnetic state of the dots. ${ }^{8,9}$ A dot with sufficiently strong magnetization and/or large enough size can create vortices or pairs of vortices in the superconductor ${ }^{10-13}$ giving rise to a variety of nontrivial commensurability effects and novel stable vortexantivortex (V-AV) configurations in superconducting films with regular arrays of magnetic dipoles (see, e.g., Refs. 14-16).

It was proposed by Carneiro ${ }^{17}$ that more control over the vortex motion can be achieved by creating a magnetic vortex ratchet, consisting of an array of in-plane magnetic dipoles. Indeed, vortices will move in one specific direction under the application of an ac excitation. Recently this magnetic ratchet effect was observed experimentally. ${ }^{18}$ In this case, however, the magnetization of the magnetic bars was sufficient to generate vortex-antivortex pairs and a ratchet signal was even detected in zero external field, implying the V-AV pairs were moving. It was argued that these vortices and antivortices annihilated in between two neighboring dipoles while new pairs were created. In this paper, we address the situation where the aforementioned annihilation mechanism for the detached vortices is not operative. We consider a superconductor-ferromagnet structure with a single in-plane magnetic dipole and investigate the vortex dynamics in the regime where the magnetic dipole periodically generates $\mathrm{V}-\mathrm{AV}$ pairs. The model used is described in Sec. II. In Sec. III we discuss the dynamics of $\mathrm{V}-\mathrm{AV}$ generation in the sys- tem under consideration and the effect of an externally applied magnetic field on this dynamics. The main conclusions are briefly described in Sec. IV.

\section{MODEL}

The structure under consideration contains a rectangular planar superconductor with lateral sizes $L_{x}$ and $L_{y}$ and thickness $d$. A rectangular magnetic bar with sizes $a_{x}, a_{y}$, and $a_{z}$ and uniform constant magnetization $\mathbf{M}$ is separated from the superconductor by an insulator with thickness $d_{1}$ (see Fig. 1), which is sufficiently large to avoid proximity effects so that there is only electromagnetic coupling between the magnetic dipole and the superconductor. An external direct current with average density $\mathbf{j}_{e}$ is applied to the superconductor along the $y$ axis.

In our treatment of the vortex dynamics in this system, we exploit the time-dependent Ginzburg-Landau (TDGL) ap-
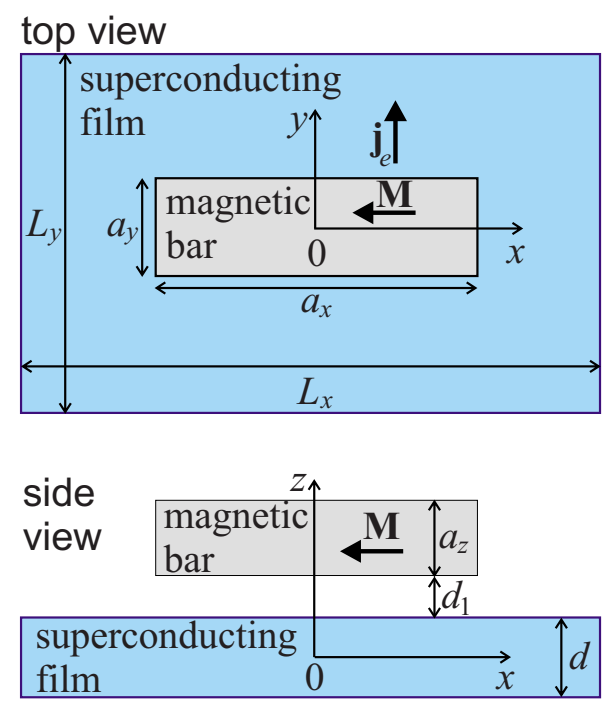

FIG. 1. (Color online) Sketch of the structure under consideration. Shaded areas correspond to the superconductor with sizes $L_{x}$, $L_{y}$, and $d$, and to the magnetic bar with sizes $a_{x}, a_{y}$, and $a_{z}$, and uniform magnetization $\mathbf{M}$. The external current with average density $j_{e}$ is applied along the $y$ axis. 
proach. We make the used variables dimensionless by expressing lengths, time, vector potential, magnetic field, magnetization, and scalar potential in units of $\sqrt{2} \xi, 2 \xi^{2} / D$, $\Phi_{0} /(2 \sqrt{2} \pi \xi), \Phi_{0} /\left(4 \pi \xi^{2}\right), \Phi_{0} /\left(4 \pi \mu_{0} \xi^{2}\right)$, and $\hbar D /\left(4 e \xi^{2}\right)$, respectively (where $\Phi_{0}$ is the magnetic-flux quantum, $\mu_{0}$ is the vacuum permeability, $\xi$ is the coherence length, and $D$ is the normal-state diffusion constant). With these units, the TDGL equation for the order parameter $\psi$ takes the form (cp., e.g., Refs. 19 and 20)

$$
\left(\frac{\partial}{\partial \tau}+i \varphi\right) \psi=(\nabla-i \mathbf{A})^{2} \psi+2 \psi\left(1-|\psi|^{2}\right),
$$

where $\varphi$ is the scalar potential and $\mathbf{A}$ is the vector potential. The scalar-potential distribution is determined from the condition $\nabla \cdot \mathbf{j}=0$, which reflects the continuity of currents in the superconductor. The used current-density unit, $\Phi_{0} c /\left(2 \sqrt{2} \pi \mu_{0} \lambda^{2} \xi\right)$, where $\lambda$ is the penetration depth, coincides with the "bulk" critical density. The current density $\mathbf{j}$ $=\mathbf{j}_{n}+\mathbf{j}_{s}$ is a sum of the normal and superconducting components, $\mathbf{j}_{n}=-(\sigma / 2)(\nabla \varphi+\partial \mathbf{A} / \partial \tau)$ and $\mathbf{j}_{s}=\operatorname{Im}\left(\psi^{*} \nabla \psi\right)-\mathbf{A}|\psi|^{2}$, where $\sigma$ is the normal conductivity, taken as $\sigma=1 / 12$ in our units. $^{20}$ The vector potential $\mathbf{A}$ can be represented as $\mathbf{A}$ $=\mathbf{A}_{M}+\mathbf{A}_{0}+\mathbf{A}_{1}$, where $\mathbf{A}_{M}$ corresponds to the magnetic field, induced by the magnetic bar, and $\mathbf{A}_{0}$ denotes the vector potential corresponding to an additional external homogeneous magnetic field $\mathbf{B}_{0}$, which may be applied to the system. The vector potential $\mathbf{A}_{1}$ describes magnetic fields, induced by the currents, which flow in the superconductor. The thickness of the superconducting layer is assumed to be sufficiently small as compared to $\xi$ and $\lambda$ in order to neglect both variations in the order-parameter magnitude across the layer and the $\mathbf{A}_{1}$ contribution. The applicability range of these assumptions will be further discussed in Sec. III. We also restrict our analysis to the case of relatively weak fields $\mathbf{B}_{0}$, which do not influence the magnetization state of the magnetic bar.

The finite-difference scheme, applied to solve the twodimensional version of Eq. (1), is similar to that reported in Refs. 20 and 21. Two-dimensional grids, used in our calculations, typically have three to ten equally spaced nodes per unit length. The step of the time variable $\tau$ is automatically adapted in the course of calculation. This adaptation is aimed to minimize the number of steps in $\tau$ and-at the same time- to keep the solving procedure stable. On average, the step in $\tau$ is $\sim 10^{-5}$ to $\sim 10^{-3}$ depending on the used grid as well as on a specific distribution of the order parameter. For each momentary distribution of the order parameter, an iteration procedure is used to determine the corresponding distribution of the scalar potential $\varphi$ with a relative accuracy not worse than $10^{-4}$. The superconductor/insulator boundary conditions $\left.\left(\partial / \partial x-i A_{x}\right) \psi\right|_{x= \pm L_{x} / 2}=0$ and $\partial \varphi /\left.\partial x\right|_{x= \pm L_{x} / 2}=0$ are assumed in the $x$ direction. In the $y$ direction, the boundary conditions are taken in the form $\left(\partial / \partial y-i A_{y}\right.$ $\left.-i j_{e} /|\psi|^{2}\right)\left.\psi\right|_{y= \pm L_{\gamma} / 2}=0$ and $\partial \varphi /\left.\partial y\right|_{y= \pm L_{\gamma} / 2}=0$, which corresponds to a uniform supercurrent density (equal to $j_{e}$ ) at $y$ $= \pm L_{y} / 2$, where the order parameter is assumed to be sufficiently large.

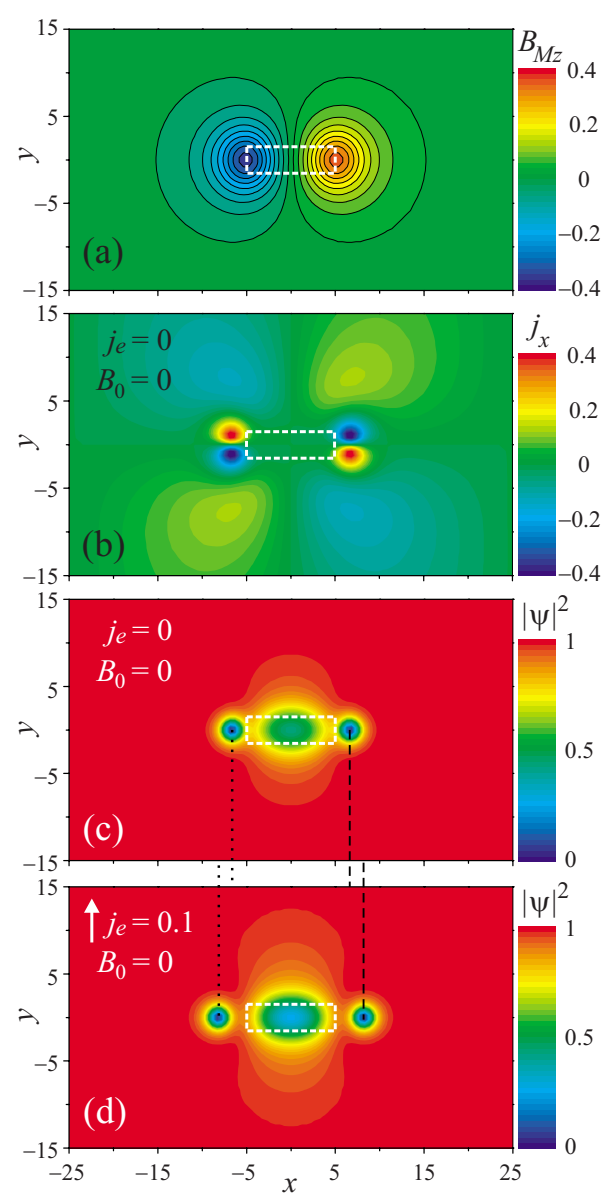

FIG. 2. (Color online) Distributions of (a) the $z$ component of the magnetic field induced by the magnetic bar in the superconducting film, (b) the $x$ component of the current density in the film at $j_{e}=0, B_{0}=0$, and the square modulus of the order parameter at $B_{0}$ $=0$ in the cases of (c) $j_{e}=0$ and (d) $j_{e}=0.1$. The parameters of the magnetic bar with shape and position, schematically shown by the white dashed line, are $a_{x}=10, a_{y}=3, a_{z}=3, d_{1}=3$, and $M_{x}=-10$. The dashed (dotted) vertical black lines in panels (c) and (d) correspond to the positions of the vortex (antivortex) core at $j_{e}=0$ and $j_{e}=0.1$.

\section{RESULTS AND DISCUSSION}

We focus on the case when the stray fields of the magnetic bar are strong enough to create $\mathrm{V}$-AV pairs in a thin superconductor film $(d=0.01$ in the calculations, presented below). As an example of such a situation we consider below a magnetic bar with $a_{x}=10, a_{y}=3, a_{z}=3, M_{x}=-10$, and $d_{1}=3$. Figure 2(a) displays the normal component of the field, induced by this magnet in the superconductor film.

As obvious from the definition of the used dimensionless parameters, one and the same set of these parameters can correspond to structures with different sizes, depending on the coherence length and the penetration depth of the superconducting material at a given temperature. For instance, in the case of an $\mathrm{Al}$ film with the zero-temperature coherence length $\xi(T=0)=87 \mathrm{~nm}$ and the penetration depth $\lambda(T=0)$ $=130 \mathrm{~nm}$ (Ref. 18) at the temperature $T=0.97 T_{c}$ the values of $a_{x}, a_{y}, a_{z}, M_{x}$, and $d_{1}$, indicated above, would correspond to a magnetic bar with sizes of about $7.1 \times 2.1 \times 2.1 \mu \mathrm{m}^{3}$, 
magnetization $5270 \mathrm{~A} / \mathrm{m}$, and the spacing between the bar and the superconductor $3.1 \mu \mathrm{m}$. In this case, the value $L_{x}$ $=L_{y}=50$, used in our calculations below, corresponds to the lateral size of the superconductor $35.3 \mu \mathrm{m}$ while the magnetic-field and current-density units are $0.66 \mathrm{mT}$ and $2.52 \times 10^{5} \mathrm{~A} / \mathrm{cm}^{2}$, respectively. The corresponding magnitude of the bar-induced magnetic fields, shown in Fig. 2(a), is about $0.25 \mathrm{mT}$. The indicated geometric parameters and magnetization of the magnetic bar are quite achievable. It is also obvious that for the described structure, variations in the order-parameter magnitude across the superconductor film can be safely neglected if the thickness of this film is smaller than $100 \mathrm{~nm}$.

Figures 2(b) and 2(c), where we plot the equilibrium distributions of the $x$ component of the current density and the order parameter at $j_{e}=0$ and $B_{0}=0$ show a vortex and an antivortex, which are nucleated by the stray field of the magnetic bar and occupy symmetric positions near the right-hand side (rhs) and left-hand side (lhs) edges of the bar, respectively. Due to the Lorentz force, produced by highly inhomogeneous stray fields of the magnet, the $\mathrm{V}$-AV pair remains pinned at densities of the externally applied current $j_{e}$ $<0.118$; those currents result only in a shift of the vortex and antivortex to new equilibrium positions at somewhat larger distances from the poles of the magnetic bar [Fig. 2(d)].

At current densities $j_{e}>0.118$, which are sufficient for detaching the vortex and antivortex from the magnetic dipole, the unpinned vortex (antivortex), driven by the Magnus force, moves along the $x$ axis toward the rhs (lhs) edge of the superconductor, where it leaves the sample [in the case of $\xi(T=0)=87 \mathrm{~nm}, \lambda(T=0)=130 \mathrm{~nm}$, and $T=0.97 T_{c}$, described above, this regime corresponds to current densities larger than $\left.3 \times 10^{4} \mathrm{~A} / \mathrm{cm}^{2}\right]$. However, new $\mathrm{V}-\mathrm{AV}$ pairs are generated by the magnetic dipole replacing those disappearing from the superconductor [see the insets for $j_{e}=0.36$ in Fig. 3(a)]. This periodic process results in a vortex flow and hence leads to a nonzero time-averaged resistance of the superconductor. The time-averaged voltage drop $\langle V\rangle$ $\equiv\left\langle\left.\varphi\right|_{x=0, y=-L_{y} / 2}-\left.\varphi\right|_{x=0, y=L_{y} / 2}\right\rangle$ as a function of the applied current density $j_{e}$ is plotted in Fig. 3(a). With increasing $j_{e}$, the generation rate of moving V-AV pairs increases [see Fig. 3 (b)], resulting in a monotonous (though nonlinear) increase in $\langle V\rangle$. In contrast to samples with lateral sizes comparable to $\xi$, where the onset of vortex motion immediately leads to the formation of a phase-slip line, ${ }^{22}$ in the case, analyzed here, a vortex-flow regime persists within rather broad range of the transport current densities. Only when increasing the current density up to $j_{e} \geq 0.383$, vortices (antivortices) in the vortex (antivortex) stream merge together and form a phase-slip line [see the inset for $j_{e}=0.4$ in Fig. 3(a)], characterized by oscillations in the voltage drop with the Josephson frequency (that is, $\omega=\langle V\rangle$ in our units). When decreasing the applied current density, starting from $j_{e}>0.383$, the phase-slip regime persists down to $j_{e} \approx 0.373$, demonstrating a hysteretic behavior typical for the phase-slip processes (see, e.g., Refs. 23 and 24).

As illustrated by Fig. 3(b), an external homogeneous magnetic field $B_{0} \neq 0$, even relatively weak as compared to the stray fields of the magnetic bar, has a pronounced effect on

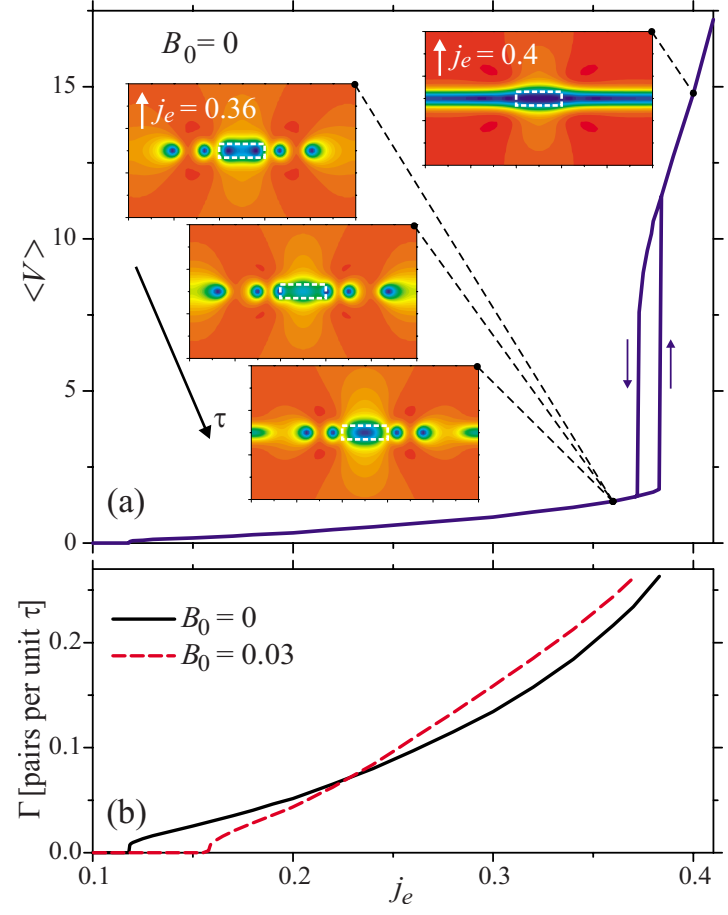

FIG. 3. (Color online) Panel (a): time-averaged voltage drop $\langle V\rangle$ as a function of the density of the externally applied current $j_{e}$ in the case of $B_{0}=0$. The vertical up (down) arrow labels the part of the curve for increasing (decreasing) applied currents. The insets show few snapshots of the order-parameter distribution, which illustrate the vortex and antivortex dynamics at $j_{e}=0.36$ and the coalescence of vortices and antivortices into a phase-slip line at $j_{e}=0.4$. The position of the magnetic bar is schematically shown by the white dashed line. Panel (b): generation rate $\Gamma$ for $\mathrm{V}-\mathrm{AV}$ pairs in the vortex-flow regime as a function of $j_{e}$ at $B_{0}=0$ (solid line) and $B_{0}$ $=0.03$ (dashed line). Both panels correspond to $L_{x}=L_{y}=50$.

the vortex generation rate $\Gamma$. On the one hand, it shifts the onset of the $\mathrm{V}-\mathrm{AV}$ emission to a higher current density as compared to the case of $B_{0}=0$. This shift is related to the interaction of the vortex, pinned at the rhs pole of the magnetic bar, with other vortices, which are present on the rhs from the bar in the equilibrium state at $B_{0}=0.03$ (see Fig. 4). Tending to push the pinned vortex toward the pole, this repulsive interaction effectively enhances pinning and hence hampers the onset of the $\mathrm{V}-\mathrm{AV}$ generation. On the other hand, at $B_{0} \neq 0$ an additional Lorenz force, proportional to $B_{0} j_{e}$, contributes to an increase in the velocity of moving vortices with increasing $j_{e}$. As a result, at $B_{0} \neq 0$ the slope of the curve $\Gamma\left(j_{e}\right)$ (as well as the differential resistance, not shown here) increases and the formation of a phase-slip line occurs at a lower current density $j_{e}$ (note that the threshold value of the generation rate, which corresponds to the formation of a phase-slip line, is practically the same for $B_{0}=0$ and $B_{0}=0.03: \Gamma \approx 0.26$ ).

A self-consistent account of the $A_{1}$ contribution to the vector potential leads (due to a partial screening of the magnetic field, induced by the magnetic bar, by supercurrents) to a reduction in the calculated generation rate of $\mathrm{V}-\mathrm{AV}$ pairs as compared to the results of the approximation $A_{1}=0$. The results of our calculations (not presented here) show that for 

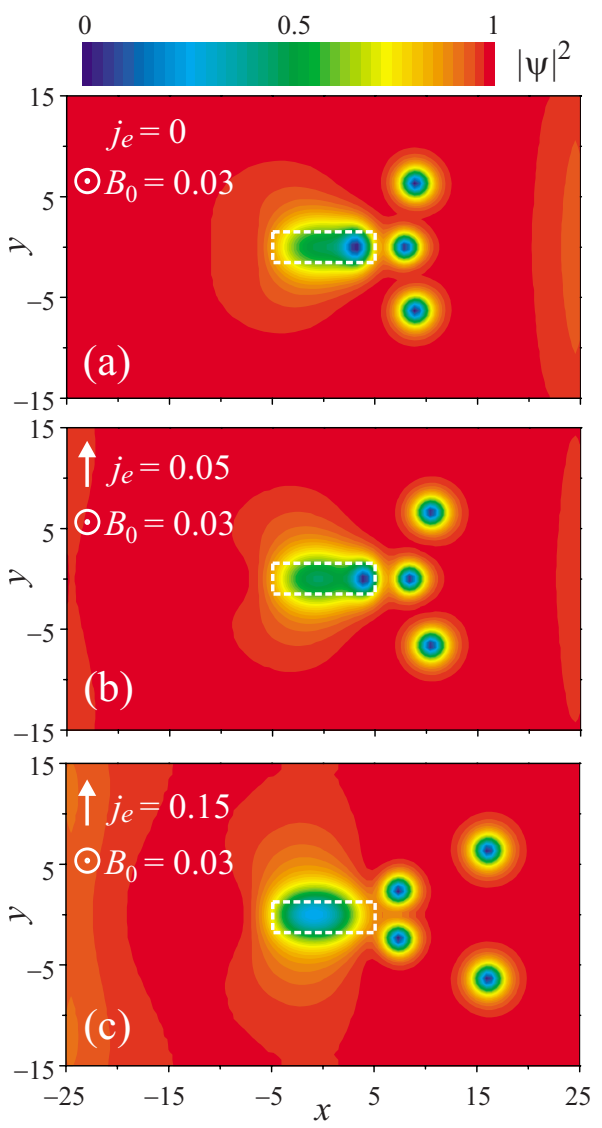

FIG. 4. (Color online) Equilibrium distributions of the square modulus of the order parameter at different densities $j_{e}$ of the externally applied current in the case of $B_{0}=0.03$. The calculations are performed for $L_{x}=L_{y}=50$ and the magnetic-bar position, schematically shown by the white dashed line.

the dimensionless thickness of the superconductor $d=0.06$ and the applied current density $j_{e}=0.3$ this reduction is about $20 \%$. Of course, in such a case the used approach, which neglects the $A_{1}$ contribution, allows only for a qualitative rather than quantitative description of the vortex-antivortex dynamics. However, the accuracy of this approach considerably increases when decreasing the thickness of the superconductor. Thus, at $d=0.012$ and $j_{e}=0.2$ the dimensionless magnetic fields related to $A_{1}$ do not exceed 0.012 (see Fig. 5). These fields are much lower than the magnitude of the bar-induced magnetic field [see Fig. 2(a)] and they do not appreciably affect the vortex dynamics: at $d=0.012$ and $j_{e}$ $=0.2$ the overestimation of the generation rate, caused by the approximation $A_{1}=0$, is less than $3.8 \%$. In the particular case of a superconductor with $\xi(T=0)=87 \mathrm{~nm}$ and $\lambda(T=0)$ $=130 \mathrm{~nm}$ at $T=0.97 T_{c}$, considered above, the fields related to the vector potential $\mathbf{A}_{1}$ are smaller than $d$ $\times 0.001 \mathrm{mT} / \mathrm{nm}$ and their influence on the vortex dynamics can be neglected for the superconductor thickness $\sim 10 \mathrm{~nm}$.

As a convenient way to represent graphically timeaveraged trajectories of vortices and antivortices, generated by the magnetic dipole, as well as the magnitude of their velocities, we calculate the spatial distribution of the parameter

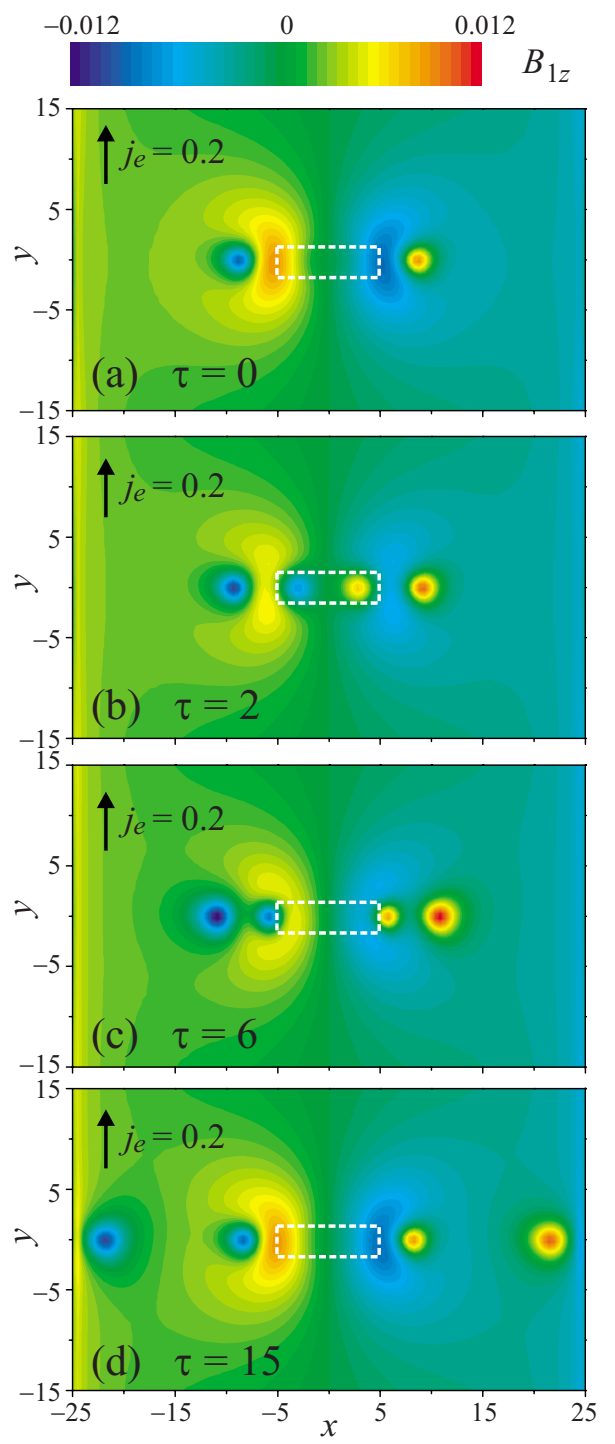

FIG. 5. (Color online) Four snapshots of the $z$ component of the magnetic field induced in the superconductor by the currents, which flow in it at $j_{e}=0.2$ and $B_{0}=0$. The time moment $\tau=0$ is chosen at random within the periodic $\mathrm{V}-\mathrm{AV}$ generation process. The calculations are performed for $L_{x}=L_{y}=50$ and the magnetic-bar position, schematically shown by the white dashed line.

$$
S(x, y)=\sqrt{\frac{1}{\tau_{2}-\tau_{1}} \int_{\tau_{1}}^{\tau_{2}} d \tau\left(\frac{\partial|\psi(x, y)|^{2}}{\partial \tau}\right)^{2}},
$$

where the integration interval $\tau_{2}-\tau_{1}$ is much larger than $\Gamma^{-1}$. The parameter $S$ takes nonvanishing values only in the regions, where the modulus of the order parameter changes with time. Those changes are especially pronounced when a moving vortex core passes through a given point $(x, y)$ and the corresponding values of $S$ increase when increasing the vortex (antivortex) velocity. As shown in Fig. 6(a), at $B_{0}=0$ vortices and antivortices, generated by the magnetic dipole, form two symmetric "streams." Since the motion of a vortex (an antivortex) is significantly accelerated when this vortex (antivortex) approaches the rhs (lhs) boundary of the superconductor, the largest values of $S$ correspond to $x$ close to 


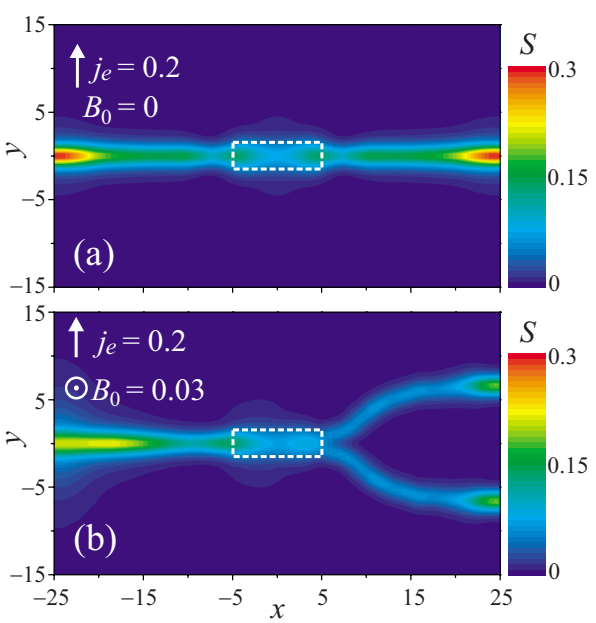

FIG. 6. (Color online) Distribution of the parameter $S$, used to visualize time-averaged trajectories of vortices and antivortices, in the cases of no homogeneous external magnetic field $B_{0}=0$ [panel (a) $]$ and $B_{0}=0.03$ [panel (b)]. The calculations are performed for $L_{x}=L_{y}=50, j_{e}=0.2$, and the magnetic-bar position, schematically shown by the white dashed line.

$\pm L_{x} / 2$. When applying an external homogeneous magnetic field, parallel to the $z$ axis, the presence of antivortices in the superconductor becomes energetically less favorable while the presence of vortices becomes more advantageous, so that moving vortices are accumulated in a vortex-rich region on the rhs from the magnetic bar. Mutual repulsion of these vortices splits the vortex stream into two branches [Fig. 6(b)]. Our calculations show that this splitting becomes weaker when increasing $j_{e}$ and that at $j_{e} \approx 0.369$ both branches coalesce into a single phase-slip line.

At sufficiently large distances between the magnetic bar and the edge of the superconductor, the presence of an additional homogeneous magnetic field can result in further bifurcation of vortex trajectories [see Fig. 7(a)]. Since new $\mathrm{V}-\mathrm{AV}$ pairs are generated by the magnetic dipole one by one and vortices have a nonzero $y$ component of velocity, symmetry with respect to the $x$ axis is inevitably broken for momentary distributions of moving vortices [see Fig. 7(b)] quite different from the rather symmetric equilibrium distributions shown in Fig. 4 and the time-averaged $S$ distribution shown in Fig. 7(a). A detailed analysis of the V-AV dynamics (not presented here) shows that in the case under consideration only a fraction of antivortices, generated by the magnetic dipole, reaches the lhs boundary of the superconductor. Other antivortices are annihilated by vortices, which enter the superconductor at $y=0$ and $x=-L_{x} / 2$ [see the distributions of $|\psi|^{2}$ and $j_{s x}$ on the lhs from the magnetic bar in Figs. 7(b) and 7(c)]. In Fig. 7(a), an accelerated motion of those vortices and antivortices toward each other manifests itself through a pronounced maximum of $S$ approximately in the middle of the distance between the magnetic bar and the lhs boundary of the superconductor.

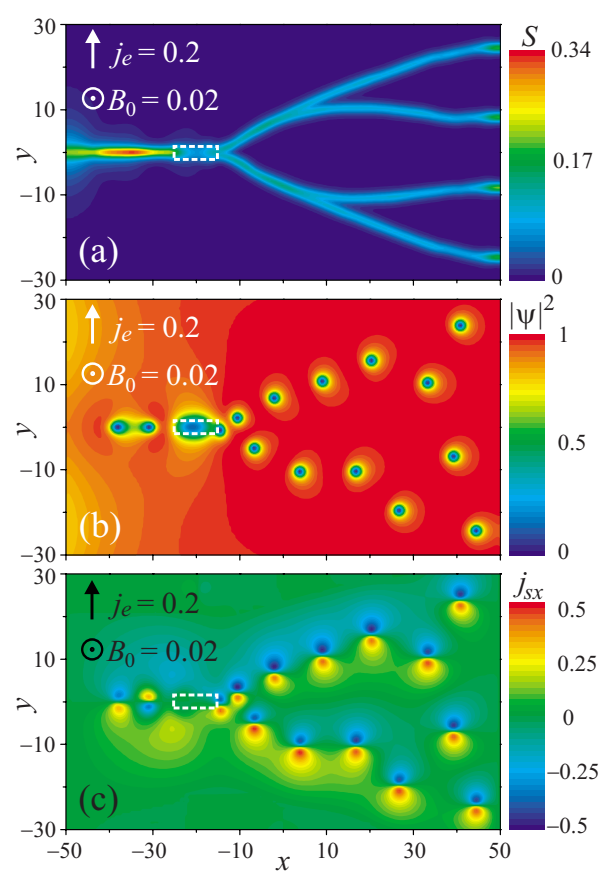

FIG. 7. (Color online) (a) Distribution of the parameter $S$ used to visualize trajectories of vortices and antivortices, (b) a snapshot of the order-parameter distribution, and (c) the corresponding distribution of the $x$ component of the supercurrent density. The density of the externally applied current is $j_{e}=0.2$ and the homogeneous external magnetic field is $B_{0}=0.02$. The calculations are performed for $L_{x}=L_{y}=100$ and the magnetic-bar position, schematically shown by the white dashed line.

\section{CONCLUSIONS}

To conclude, we have used the TDGL approach to describe a vortex-antivortex generator, formed by adding an in-plane magnetic dipole to a superconducting film. This regime of vortex-antivortex generation can be realized for a relatively broad range of transport current densities, limited on the one hand by $\mathrm{V}-\mathrm{AV}$ pinning and on the other hand by the formation of a phase-slip line. We have shown that the generation rate as well as the trajectory patterns of emitted vortices are highly sensitive not only to the applied transport current but also to the presence of an additional weak homogeneous magnetic field, which leads to a faster increase in the generation rate with increasing current and to a splitting of the stream of emitted vortices into two or more branches.

\section{ACKNOWLEDGMENTS}

This work is supported by the Methusalem funding of the Flemish Government, the NOI BOF UA 2004, the FWO-V under Projects No. G.0435.03, No. G.0356.06, No. G.0115.06, No. G.0180.09N, and No. G.0370.09, the IAP network of the Belgian Government, and by the ESF-NES Programme. W.G. is grateful for the support from FWOVlaanderen. 
${ }^{1}$ I. F. Lyuksyutov and V. L. Pokrovsky, Adv. Phys. 54, 67 (2005).

${ }^{2}$ A. I. Buzdin, Rev. Mod. Phys. 77, 935 (2005).

${ }^{3}$ A. Yu. Aladyshkin, A. V. Silhanek, W. Gillijns, and V. V. Moshchalkov, Supercond. Sci. Technol. 22, 053001 (2009).

${ }^{4}$ J. I. Martín, M. Vélez, J. Nogués, and I. K. Schuller, Phys. Rev. Lett. 79, 1929 (1997).

${ }^{5}$ D. J. Morgan and J. B. Ketterson, Phys. Rev. Lett. 80, 3614 (1998).

${ }^{6}$ M. J. Van Bael, K. Temst, V. V. Moshchalkov, and Y. Bruynseraede, Phys. Rev. B 59, 14674 (1999).

${ }^{7}$ M. J. Van Bael, J. Bekaert, K. Temst, L. Van Look, V. V. Moshchalkov, Y. Bruynseraede, G. D. Howells, A. N. Grigorenko, S. J. Bending, and G. Borghs, Phys. Rev. Lett. 86, 155 (2001).

${ }^{8}$ M. Lange, M. J. Van Bael, Y. Bruynseraede, and V. V. Moshchalkov, Phys. Rev. Lett. 90, 197006 (2003).

${ }^{9}$ W. Gillijns, A. V. Silhanek, V. V. Moshchalkov, Phys. Rev. B 74, 220509(R) (2006).

${ }^{10}$ I. F. Lyuksyutov and V. I. Pokrovsky, Phys. Rev. Lett. 81, 2344 (1998).

${ }^{11}$ S. Erdin, A. F. Kayali, I. F. Lyuksyutov, and V. L. Pokrovsky, Phys. Rev. B 66, 014414 (2002).

${ }^{12}$ M. A. Kayali, Phys. Rev. B 69, 012505 (2004).
${ }^{13}$ S. Erdin, Phys. Rev. B 72, 014522 (2005).

${ }^{14}$ M. V. Milošević and F. M. Peeters, Phys. Rev. Lett. 93, 267006 (2004).

${ }^{15}$ D. J. Priour, Jr. and H. A. Fertig, Phys. Rev. Lett. 93, 057003 (2004).

${ }^{16}$ M. V. Milošević and F. M. Peeters, Physica C 437-438, 208 (2006).

${ }^{17}$ G. Carneiro, Physica C 432, 206 (2005).

${ }^{18}$ C. C. de Souza Silva, A. V. Silhanek, J. Van de Vondel, W. Gillijns, V. Metlushko, B. Ilic, and V. V. Moshchalkov, Phys. Rev. Lett. 98, 117005 (2007).

${ }^{19}$ C. R. Hu and R. S. Thomson, Phys. Rev. B 6, 110 (1972).

${ }^{20}$ R. Kato, Y. Enomoto, and S. Maekawa, Phys. Rev. B 44, 6916 (1991).

${ }^{21}$ V. N. Gladilin, J. Tempere, I. F. Silvera, J. T. Devreese, and V. V. Moshchalkov, Phys. Rev. B 77, 024512 (2008).

${ }^{22}$ M. V. Milošević, G. R. Berdiyorov, and F. M. Peeters, Phys. Rev. Lett. 95, 147004 (2005).

${ }^{23}$ M. Tinkham, Introduction to Superconductivity, 2nd ed. (McGraw-Hill, New York, 1996).

${ }^{24}$ B. I. Ivlev and N. B. Kopnin, Adv. Phys. 33, 47 (1984). 